\title{
The effect of UV-B on Arabidopsis leaves depends on light conditions after treatment
}

\author{
Olga Sztatelman ${ }^{1,2}$, Joanna Grzyb ${ }^{3}$, Halina Gabryś ${ }^{1}$ and Agnieszka Katarzyna Banaś , $^{*}$ (D)
}

\begin{abstract}
Background: Ultraviolet B (UV-B) irradiation can influence many cellular processes. Irradiation with high UV-B doses causes chlorophyll degradation, a decrease in the expression of genes associated with photosynthesis and its subsequent inhibition. On the other hand, sublethal doses of UV-B are used in post-harvest technology to prevent yellowing in storage. To address this inconsistency the effect of short, high-dose UV-B irradiation on detached Arabidopsis thaliana leaves was examined.

Results: Two different experimental models were used. After short treatment with a high dose of UV-B the Arabidopsis leaves were either put into darkness or exposed to constant light for up to 4 days. UV-B inhibited dark-induced chlorophyll degradation in Arabidopsis leaves in a dose-dependent manner. The expression of photosynthesis-related genes, chlorophyll content and photosynthetic efficiency were higher in UV-B -treated leaves left in darkness. UV-B treatment followed by constant light caused leaf yellowing and induced the expression of senescence-related genes. Irrespective of light treatment a high UV-B dose led to clearly visible cell death 3 days after irradiation.
\end{abstract}

Conclusions: High doses of UV-B have opposing effects on leaves depending on their light status after UV treatment. In darkened leaves short UV-B treatment delays the appearance of senescence symptoms. When followed by light treatment, the same doses of UV-B result in chlorophyll degradation. This restricts the potential usability of UV treatment in postharvest technology to crops which are stored in darkness.

Keywords: Cell death, Chlorophyll degradation, Light, Photosynthesis, Senescence, UV-B

\section{Background}

Beside visible light the solar radiation which strikes the Earth's atmosphere also contains ultraviolet (UV) and infrared irradiation. Based on the biological effects it induces, UV is divided into UV-C (100-280 nm), UV-B $(280-320 \mathrm{~nm})$ and UV-A (320-400 nm). UV-C, the most dangerous, is completely absorbed by the ozone layer in the atmosphere. As a consequence, UV-B is the shortest wavelength component of the sunlight which reaches the surface of the Earth. As an integral part of

\footnotetext{
* Correspondence: a_katarzyna.banas@uj.edu.pl

${ }^{1}$ Department of Plant Biotechnology, Faculty of Biochemistry, Biophysics and Biotechnology, Jagiellonian University, Gronostajowa 7, Krakow 30-387,

Poland

${ }^{4}$ The Malopolska Centre of Biotechnology, Jagiellonian University, Gronostajowa 7, Krakow 30-387, Poland

Full list of author information is available at the end of the article
}

solar radiation, UV always accompanies visible light. This is of special importance for plants which are both sessile and photosynthetic organisms. The UV-B range is absorbed by many constituents of the cell with harmful consequences. UV-B is cytotoxic, damaging the cell at many levels, including nucleic acids, lipids, photosynthetic pigments and proteins [1]. Higher levels of UV-B cause the production of reactive oxygen species (ROS) and activate general stress signaling pathways [2]. Moreover, the UV-B-dependent formation of dimers between adjacent pyrimidines in DNA strands may be both mutagenic and genotoxic due to blocking the progress of DNA polymerase. As a result, the exposure of plants to high levels of UV can lead to cell death dependent on ROS signaling (for a review see: [3]).

The depletion of the ozone layer has resulted in an increase in the level of UV-B reaching the Earth's surface. 
That is why the impact of this wavelength range on living organisms started to be intensively investigated in the eighties. Most experiments have been performed in growth chambers with relatively low photosynthetically active radiation (PAR) supplemented with a high dose of UV-B [4]. They showed a very strong impact of UV-B on the content of photosynthesis dependent pigments, the activity of photosynthetic enzymes and photosynthetic efficiency (for a review see: [5]). UV-B affects Photosystem II (PSII) to much greater extent than Photosystem I [1]. The degradation of integral components of PSII reaction centers, including D1 and D2 proteins, is an extensively studied aspect of the effect of UV-B on photosynthesis. Many compounds have been hypothesized as primary targets of UV-B action on photosynthesis, including the reaction centre itself, quinone acceptors and redoxactive tyrosines [1]. UV-B is also absorbed by the oxygenevolving Mn cluster and can cause its damage [6].

The reaction to UV depends both on its dose and the irradiation scheme. Acute treatment has a more severe effect than chronic exposure which activates acclimation responses $[7,8]$. These responses are aimed at minimizing the impact of UV-B on plant cells. They include leaf thickening, alterations in cuticular wax layers and the biosynthesis of UV-B-absorbing phenolic compounds, such as flavonoids [5]. Leaf yellowing is one of the most visible symptoms of irradiation with high doses of UV-B. It results from chlorophyll degradation and the decreased expression of photosynthesis-related genes. There are similar symptoms during many abiotic and biotic stresses, as well as during natural senescence [9-11]. Many of the stress conditions which cause leaf yellowing also induce the expression of senescence-associated genes (SAGs) [12]. Indeed, UV-B treatment of mature leaves markedly up-regulates the expression of these genes and downregulates some photosynthesis-related genes $[13,14]$. The influence of UV irradiation on plants also depends on their age. DNA damage, measured by homologous recombination events, is clearly more severe in younger Arabidopsis [15] and Nicotiana plants than in older ones [7]. On the other hand, the decline in anthocyanin, chlorophyll and carotenoid contents as well as in photosynthetic yield is higher in older plants $[16,17]$.

The effect of UV-B on plant functioning is also affected by environmental conditions (for a review see: [18]). The negative impact of UV irradiation on the growth parameters of cucumber increased with increasing nitrogen fertilization [19]. Arabidopsis plants grown in an elevated temperature are more sensitive to UV-B irradiation [20].

There is often a synergistic effect between stresses induced by different factors. Pre-treatment of barley seedlings with other stressors, like a high $\mathrm{NaCl}$ concentration minimized the UV-B-induced decrease in the content of photosynthetic pigments and in photosynthetic efficiency [21]. After UV-B pretreatment, plant survival was enhanced under biotic and abiotic stress conditions. Plant tolerance to cold is increased by UV-B as shown by studies both in a growth chamber and in the field [15, 22]. Drought stress also has a lesser impact on plants pretreated with UV-B [23] (for a review see: [24]). UV-B irradiation can enhance plant resistance to pathogen attack via changing plant morphology, the production of secondary metabolites and the expression of genes controlling pathogen viability [25]. On the other hand, rice plants overexpressing WRKY89, a gene induced by pathogen attack, are more resistant to UV-B [26].

The interplay between PAR and UV irradiation is the most widely studied (for a review see: [27]). High light up-regulates the expression of the genes involved in flavonoids synthesis including PHENYLALANINE AMMONIALYASE 1 (PAL1) and CHALCONE SYNTHASE (CHS), as well as the genes encoding ROS scavengers [28]. Flavonoids such as isoflavons and anthocyanins are UV absorbing pigments shown to increase plant tolerance to strong UV irradiation [29]. Arabidopsis plants with an impaired production of ascorbate, a ROS scavenger, are more sensitive to UV-B [30]. This suggests that enhanced ascorbate synthesis helps plants to cope with UV-Binduced stress. The resistance of bean plants to elevated UV-B irradiation positively correlates with light intensity [31, 32]. A low dose of UV-B, when supplemented with visible light, does not influence photosynthesis or the expression of photosynthesis-related genes [33]. The chlorophyll content in plants grown in a UV-B-enriched environment may be even $25 \%$ higher than that of the control [34]. The recovery of photosynthesis after UV-B damage is also faster under illumination with photosynthetically active light [35]. UV-B causes the degradation of $\mathrm{D} 1$ protein to a $20 \mathrm{kDa}$ fragment which is subsequently completely degraded by proteases in a light-dependent manner. Additionally, de novo synthesis of D1 protein occurs only under visible light [35]. Growing plants under visible light supplemented with UV-B activates mechanisms which allow them to survive under subsequent high light stress [36].

Although high doses of UV-B have a negative impact on photosynthetic systems, UV-B is used in post-harvest technology to slow down yellowing during storage $[37,38]$. To address this inconsistency we examined the effect of short, $5 \mathrm{~min}$ high-dose $\left(8 \mathrm{~W} \cdot \mathrm{m}^{-2}\right) \mathrm{UV}-\mathrm{B}$ irradiation on detached Arabidopsis thaliana leaves. As light is known to alleviate the effects of UV-B on plants, two different experimental regimes were applied after irradiation. The irradiated samples were kept either in darkness or under constant white light for up to 4 days. To characterize the influence of UV-B on photosynthesis the content of photosynthetic pigments, levels of D1 
protein as well as photosynthetic efficiency were analyzed. The production of anthocyanins was examined both at the levels of gene expression and anthocyanin accumulation. Additionally, the expression of the senescence-associated genes, SAG12, SAG13, SENESCENCE1 (SEN1) and WRKY53 was tested. Finally, cell death was checked using trypan blue staining. The results clearly showed that irradiation with a high dose of UV-B can induce two different pathways. The key controlling factor is the presence or absence of visible light after UV-B irradiation.

\section{Methods}

\section{Plant material}

Arabidopsis thaliana Columbia-0, and uvr8-6 (uvb-resistance 8-6, SALK_033468, [39]) and mcp2d-1 (metacaspase 2d-1, SAIL_856_D05, [40]) seeds were obtained from The Nottingham Arabidopsis Stock Centre (NASC, Nottingham, UK). Mutant plants were identified by PCR analysis according to standard protocol [41] using Lba1 and gene specific primers listed in Additional file 1: Table S1.

Seeds were sown in Jiffy- $7^{\circ}$ Peat Pellet (Jiffy International AS, Kristiansand, Norway) and stratified for 2 days at $4{ }^{\circ} \mathrm{C}$. Plants were grown in a growth chamber (Sanyo MLR $350 \mathrm{H}$, Japan) at $23{ }^{\circ} \mathrm{C}, 80 \%$ relative humidity, with a 10/14 light/dark cycle and fluorescent lamps (FL40SS.W/37, Sanyo) as a light source with a photosynthetic photon flux density of $70 \mu \mathrm{mol} \cdot \mathrm{m}^{-2} \cdot \mathrm{s}^{-1}$. Adult leaves from 5-6 week old Arabidopsis plants were used for all experiments.

\section{UV-B treatment}

Two experimental models were used involving either dark or continuous light treatment. Leaves meant for dark treatment were taken from plants dark-adapted for $16 \mathrm{~h}$ prior to the experiment and handled in green safe light. Leaves meant for light treatment were taken directly from the growth chamber during the light period. The irradiation of both kind of samples started at 10 a.m. i.e. $2 \mathrm{~h}$ after the photoperiodic light had been turned on. Just before irradiation the Arabidopsis thaliana leaves were detached from the plant and put on watersoaked paper. One half of each leaf was covered with black paper (control) and the whole leaf was exposed to $5 \mathrm{~min}$ of high intensity UV-B $\left(8 \mathrm{~W} \cdot \mathrm{m}^{-2}\right.$, under USHIO UV-B Lamps G8T5E). After treatment the covers were removed and leaves were transferred either to constant darkness or to constant white light $\left(100 \mu \mathrm{mol} \cdot \mathrm{m}^{-2} \cdot \mathrm{s}^{-1}\right)$ delivered by LEDs (Tops 10 Power Pure White Led OSW4XAHAE1E). After the specified time period leaves were cut into halves and the control and treated halves from 4 different leaves were pooled together, immediately frozen in liquid nitrogen, weighed and kept at $-80{ }^{\circ} \mathrm{C}$ until further analysis. Each measurement was repeated at least 3 times. Day 0 refers to samples collected $1 \mathrm{~h}$ after UV-B treatment. The scheme of the experiment is summarized in Fig. 1.

\section{Chlorophyll Fluorescence Measurements}

Chlorophyll fluorescence in the leaves was imaged using an Open FluorCam FC 800-O/1010 imaging fluorometer (Photon Systems Instruments). Before measurements the leaves were dark-adapted for at least $30 \mathrm{~min}$. The basal fluorescence (F0) was recorded for $5 \mathrm{~s}$, followed by a $1 \mathrm{~s}$ pulse of saturating white light $\left(2000 \mu \mathrm{mol} \cdot \mathrm{m}^{-2} \cdot \mathrm{s}^{-1}\right)$. Data points represent the means of at least 12 leaf halves in 3 independent replicates.

\section{Pigment extraction}

Frozen leaf material was ground in a mortar with $0,5 \mathrm{ml}$ methanol on ice, the extract was collected and the mortar and pestle were washed with an additional $1 \mathrm{ml}$ of methanol. The extract was centrifuged with a table-top centrifuge at $14000 \mathrm{~g}$ for $1 \mathrm{~min}$. The supernatant was transferred to a new tube and the pellet was re-extracted with $0,5 \mathrm{ml}$ methanol twice. All supernatants were combined together and used for the HPLC analysis of photosynthetic pigments. Pellets were extracted on ice with $1 \mathrm{ml}$ of $0,1 \% \mathrm{HCl}$ in methanol, centrifuged and reextracted twice with $0,5 \mathrm{ml}$ of acidic methanol. The supernatants were combined together and their absorption

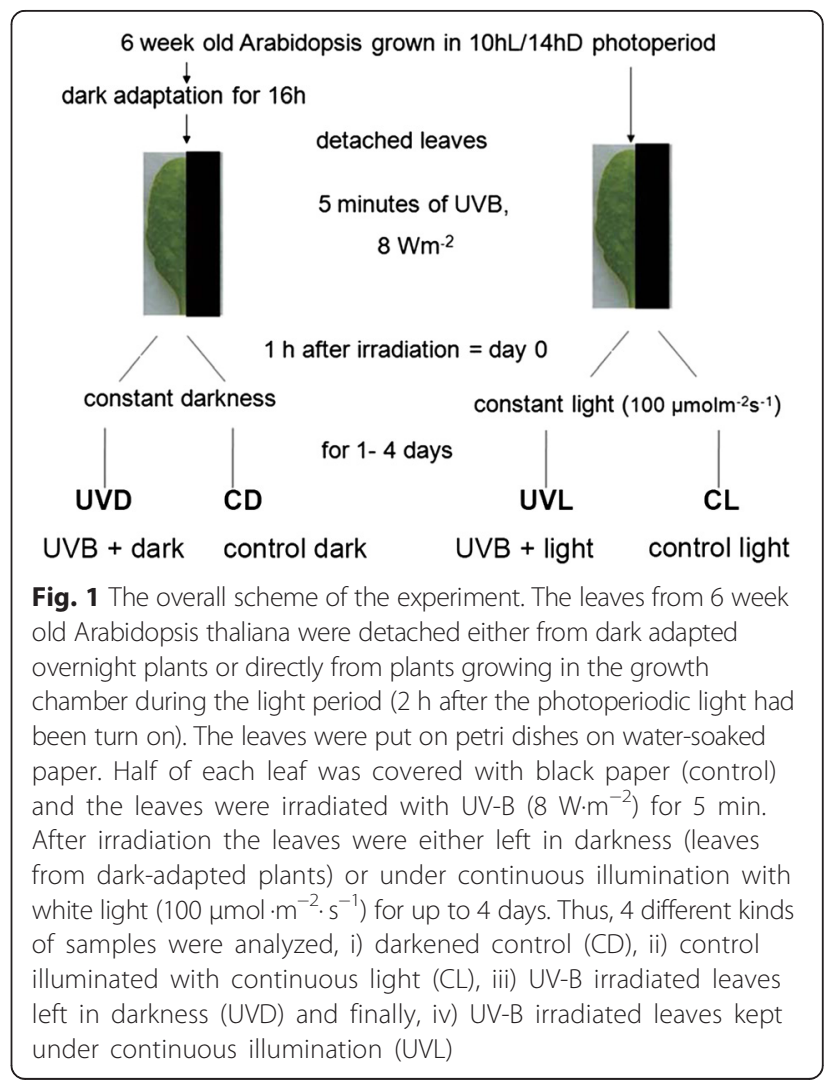


spectra were measured. Anthocyanin content was inferred from absorbance at $532 \mathrm{~nm}$.

\section{HPLC measurement}

HPLC analysis of pigments was done by a method modified from [42].100 $\mu \mathrm{l}$ of methanol pigment extract was loaded with a loop onto a C-18 column (Bionacom Velocity, 5uicrons, $4.6 \times 250 \mathrm{~mm}$ ), connected to an Akta Purifier (GE Healthcare). The column was preequilibrated with $5 \mathrm{ml}$ of solvent A (90\% acetonitrile, $10 \%$ water), and elution was done with following gradient with solvent B (100 \% ethyl acetate):

1. $1-5 \mathrm{ml}, 100 \% \mathrm{~A}$ to $80 \% \mathrm{~A}$

2. $5-20 \mathrm{ml}, 80 \% \mathrm{~A}$ to $50 \% \mathrm{~A}$

3. $20-25 \mathrm{ml}, 50 \%$ A to $30 \% \mathrm{~A}$

4. $25-30 \mathrm{ml}, 30 \% \mathrm{~A}$ (isocratic)

5. $40-45 \mathrm{ml}, 30 \%$ A to $100 \% \mathrm{~A}$.

The flow rate was $1 \mathrm{ml} / \mathrm{min}$. Elution was monitored spectrophotometrically at three wavelengths simultaneously (405 nm, $436 \mathrm{~nm}$ and $280 \mathrm{~nm}$ ). Pigments were identified by retention time, compared to standards. The chromatogram analysis and peak integration were done using Unicorn software (GE Healthcare).

For a qualitative determination of pigments, extinction coefficients in HPLC (Additional file 2: Table S2) solvents were determined as follows. Fractions corresponding to pigments of interest were collected separately in a known volume. After recording the spectra in the HPLC solvent, the fractions were dried and resuspended respectively in $80 \%$ acetone-chlorophyll a, chlorophyll b [43], methanol-violaxanthin, lutein [44], ethanol-neoxanthin [45] and hexane- $\beta$-carotene [46].

The statistical significance of the differences between treatments was assessed with one-way ANOVA, using GraphPad InStat Software (Additional file 3: Table S3).

\section{RNA isolation and real-time PCR}

RNA isolation, cDNA synthesis and real-time RT-PCR reactions were performed as given elsewhere [47]. All reactions were run in triplicates. The sequence of the primers and their annealing temperatures are listed in Additional file 1: Table S1. A single dark-adapted overnight control sample from day 0 was used as the reference for calculating relative expression levels. The normalization was performed with normalization factors based on the reference gene levels calculated by geNorm v3.4 [48].

\section{Protein extraction and Western Blot}

The leaf material was ground in liquid nitrogen. An extraction buffer (4\% SDS, $2 \% \beta$-mercaptoethanol, $2 \mathrm{mM}$ PMSF, $100 \mathrm{mM}$ TrisHCl, $\mathrm{pH} \mathrm{8,8)} \mathrm{was} \mathrm{added} \mathrm{in} \mathrm{the}$ proportion of $10 \mu \mathrm{l}$ of extraction buffer per $1 \mathrm{mg}$ of powder mass. The samples were vortexed vigorously, incubated at $80{ }^{\circ} \mathrm{C}$ for $3 \mathrm{~min}$, centrifuged for $10 \mathrm{~min}$ at 16 $000 \mathrm{~g}$ at $4{ }^{\circ} \mathrm{C}$ and supernatant was mixed with an SDSPAGE loading buffer. The SDS-PAGE was performed according to [49] in a gel containing $12 \%$ polyacrylamide using the Mini Protean system (Bio-Rad). After separation the proteins were either stained with Coomassie Brilliant Blue staining (for total protein visualization) or transferred to a PVDF membrane (ImmobilonP, Millipore) by the semi-dry transfer method (Trans-Blot SD Semi-Dry Transfer Cell, Bio-Rad) for Western Blot analysis. Membranes were stained with Ponceau $\mathrm{S}$ to ensure proper transfer, blocked with $5 \%$ fat free dried milk in PBS with 0,5\% Tween and incubated with an anti-D1 antibody (AS05 084, Agrisera) diluted 1:10 000 for $1 \mathrm{~h}$ at room temperature, followed by secondary antibody incubation (Goat anti-rabbit IgG HRP conjugated, Agrisera) under the same conditions. After that a chemiluminescence substrate was added (Clarity Western ECL Substrate, Bio-Rad) and the chemiluminescence was imaged using the BioSpectrum imaging system (UVP).

\section{Trypan Blue staining}

The samples were pretreated (i.e. kept in darkness or left for $2 \mathrm{~h}$ under photoperiodic light in the growth chamber), irradiated and kept in either darkness or constant light as described in the "UV-B treatment" section. The only exception was that prior to the irradiation, instead of leaf halves, a middle, narrow part of the detached leaf (perpendicular to the vasculature) was covered with black paper. After the specified time the leaves were covered with $2,5 \mathrm{mg} / \mathrm{ml}$ Trypan Blue in lactophenol, heated in a boiling water bath for $1 \mathrm{~min}$, stained at room temperature for an additional $2 \mathrm{~h}$, and destained with a saturated chloral hydrate solution.

\section{Results}

\section{Effect of UV-B on dark-induced yellowing of Arabidopsis} leaves

Different UV-B doses were applied in order to check whether UV-B irradiation can slow down the onset of dark-induced senescence in darkened Arabidopsis leaves. Whereas in the non-irradiated leaf halves visible symptoms of senescence, i.e. yellowing, were easy to observe (Fig. 2), UV-B treatment clearly influenced chlorophyll degradation in a dose-dependent manner. Differences in leaf color between the irradiated and non-irradiated halves started to be visible after $3 \mathrm{~min}$ of the UV-B $\left(8 \mathrm{~W} \cdot \mathrm{m}^{-2}\right)$ treatment and persisted up to the 10th minute of irradiation. Based on the results of this preliminary experiment, we decided on a 5 min treatment for further analysis. 


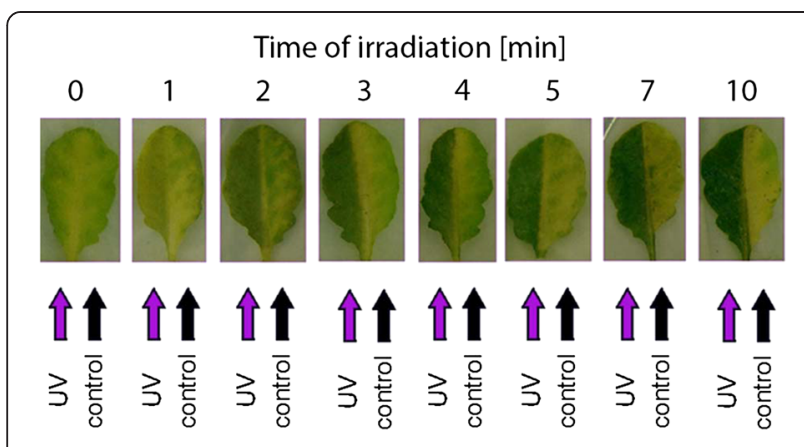

Fig. 2 Photographs of the detached leaves of 6-week old A. thaliana with one half covered with black paper, and another half irradiated with UV-B $\left(8 \mathrm{~W} \cdot \mathrm{m}^{-2}\right)$ for the indicated time and left in darkness for 4 days

The core idea of the study was to compare the effects of UV-B in dark and light conditions and that was kept in mind when setting up experimental treatments. On the one hand, we wanted to avoid possible effects of the circadian clock. On the other hand, we wanted to test the influence of UVB on either the dark- or lightadapted state of the leaves. Therefore, we decided to start both light and dark experiments at the same time point i.e. $2 \mathrm{~h}$ after dawn. In consequence, the plants used for testing the dark-adapted state were kept in darkness for that time. To make sure that this extended night did not result in drastic changes in the observed phenomena, leaf yellowing was observed in leaves taken from plants which were either dark-adapted or kept in photoperiodic light for $2 \mathrm{~h}$ before UV-B irradiation, and transferred to darkness afterwards. In both cases chlorophyll degradation was lower in UV-B irradiated leaf halves (Fig. 3, compare a and b), with differences observed only in the rate of degradation which was more prominent in control leaf halves from dark-adapted plants. Thus, for further experiments on the UV-inhibition of darkinduced chlorophyll degradation only dark-adapted plants were used (see below).

\section{Macroscopic appearance of leaves under different post- treatment light conditions}

Two different experimental models were used (Fig. 1). The first of these involved detached leaves from darkadapted plants. The leaves were UV-B irradiated and kept in darkness for up to 4 days. In the other model leaves were taken from plants $2 \mathrm{~h}$ after the start of the light period. They were UV-B irradiated and placed in constant light $\left(100 \mu \mathrm{mol} \cdot \mathrm{m}^{-2} \cdot \mathrm{s}^{-1}\right)$. Dark-induced leaf yellowing was observed in control leaf halves, while those from constant light stayed green but showed reddening, probably due to anthocyanin accumulation (Fig. 3). The opposite effect of post-UV-treatment light conditions was observed in leaf halves irradiated with $8 \mathrm{~W} \cdot \mathrm{m}^{-2}$ of UV-B for $5 \mathrm{~min}$. In irradiated leaf halves kept in

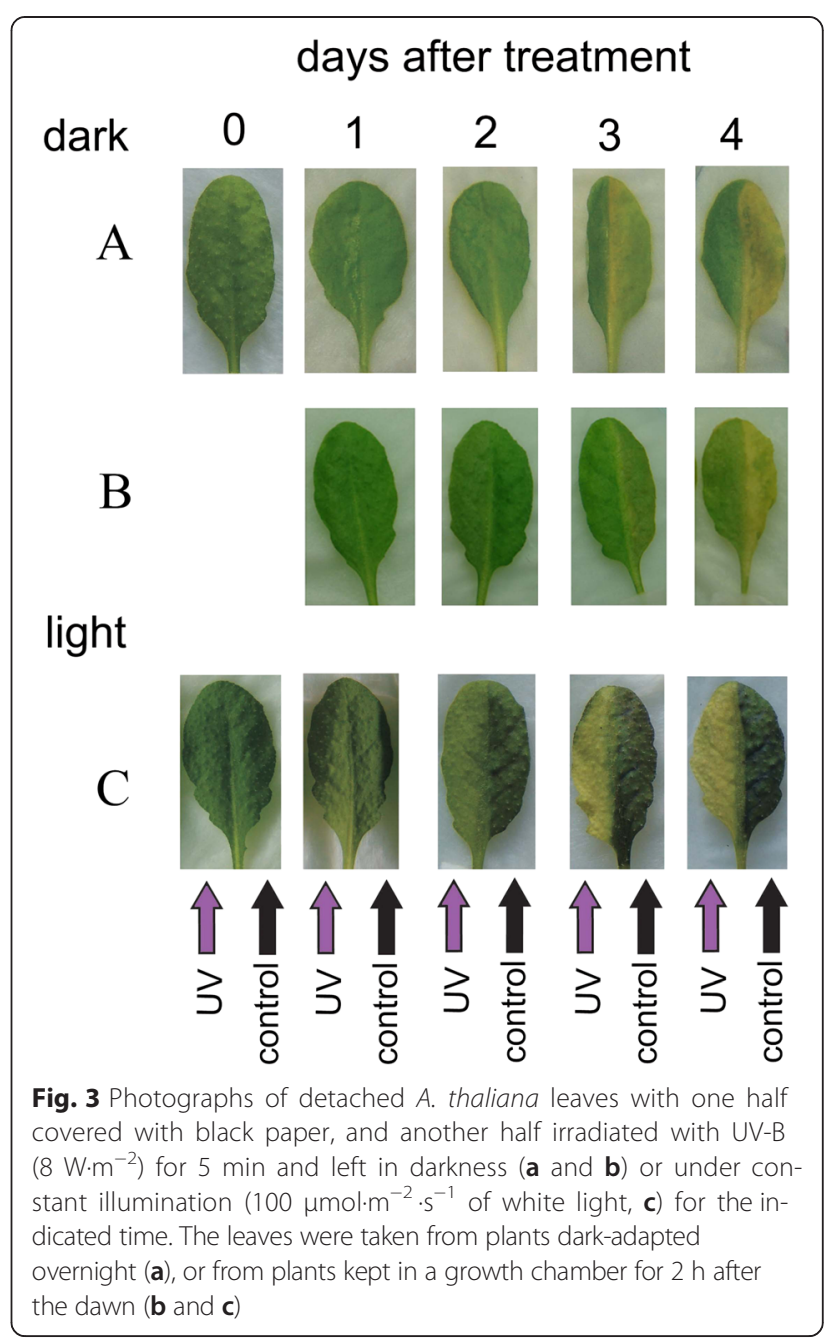

darkness dark-induced chlorophyll degradation was alleviated and yellowing was barely visible even after 4 days. In contrast, leaf halves subjected to UV-B treatment and then transferred to continuous light showed yellowing without the appearance of red coloring. To examine the observed effect in detail different parameters including chlorophyll fluorescence, the expression of senescenceinduced and photosythesis-related genes as well as the level of photosynthetic pigments and anthocyanins were investigated.

\section{Photosynthetic efficiency and photosynthetic pigment content}

To analyze the changes in pigment composition of the leaves, HPLC analysis of isolated photosynthetic pigments starting from day 0 to day 4 after UV-B treatment was carried out. The results are shown in Fig. 4a and b. The overall changes in the levels of chlorophyll a (chl a) and chlorophyll b (chl b) were similar. In continuous light, starting from the second day, the chlorophyll levels began to drop in the UV-B followed by continuous light 

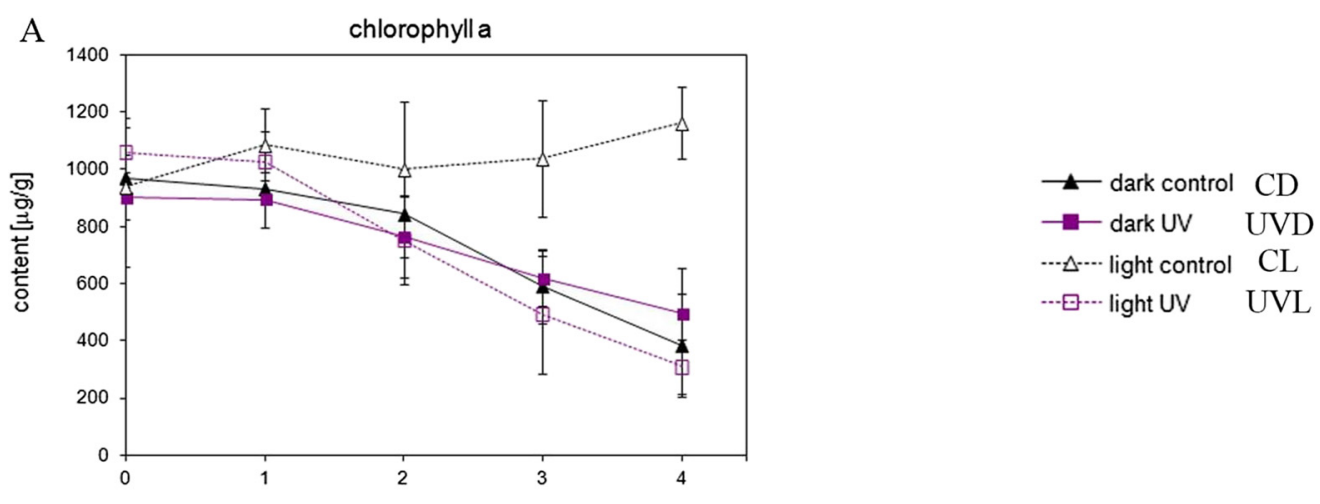

B

chlorophyll b

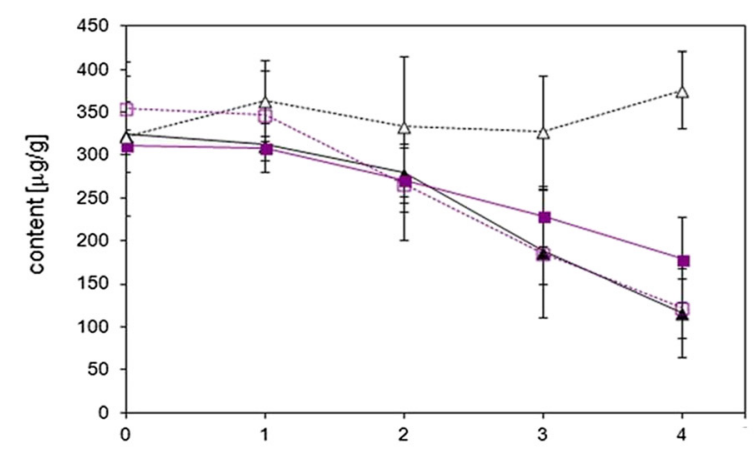

C

chlorophyll a/b

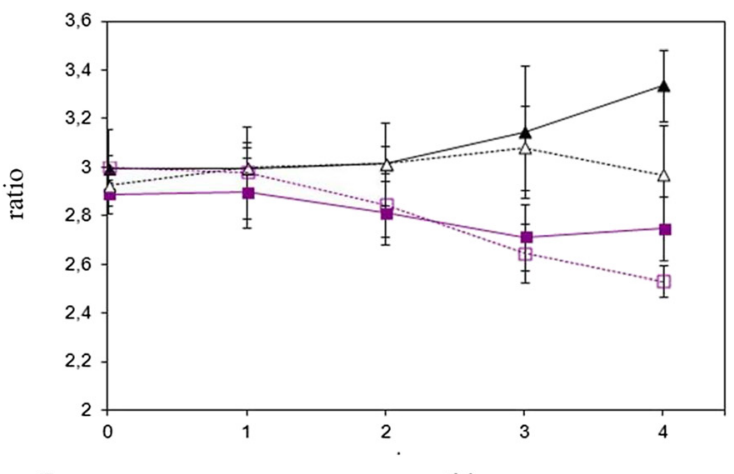

$\mathrm{D}$

violaxanthin

E

neoxanthin
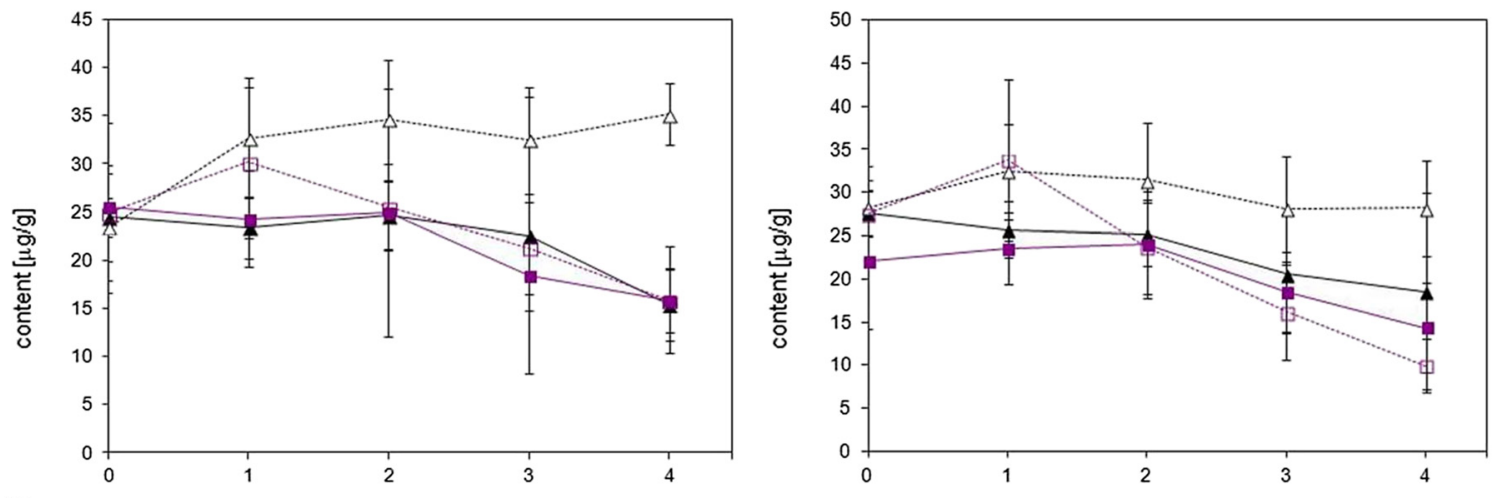

F

lutein

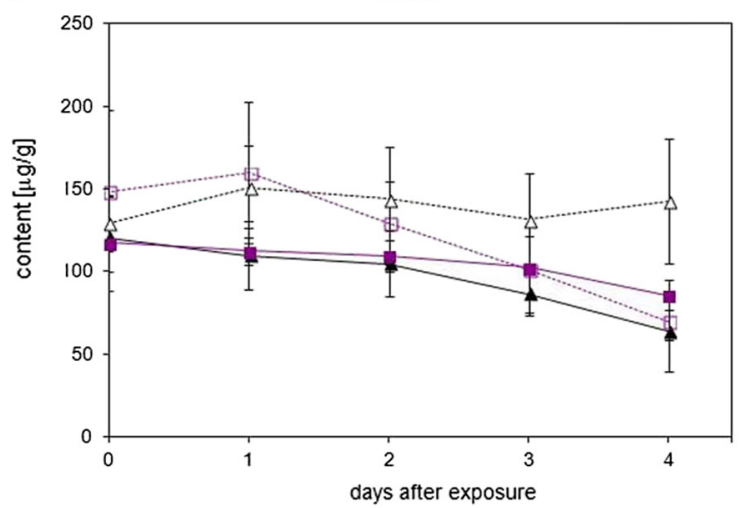

G

$\beta$-carotene

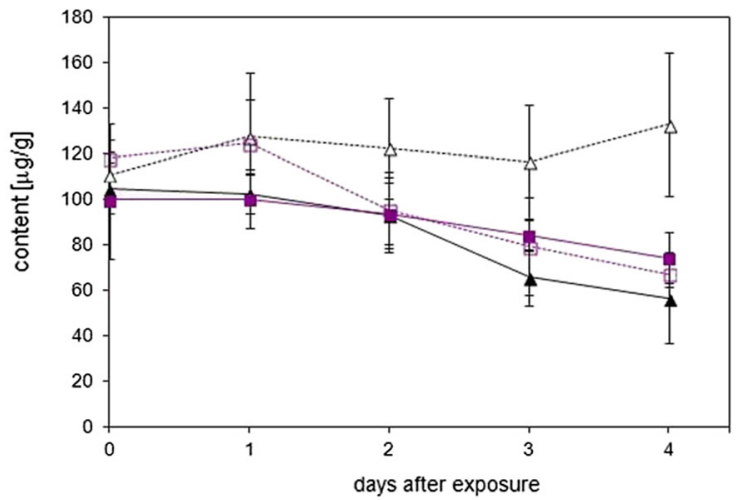

Fig. 4 (See legend on next page.) 
(See figure on previous page.)

Fig. 4 Changes in photosynthetic pigments (a chlorohyll a, b chlorophyll b, c chlorophyll a/b, $\mathbf{d}$ violaxanthin, e neoxanthin, $\mathbf{f}$ lutein, $\mathbf{g} \beta$-carotene ) in detached Arabidopsis leaf halves either irradiated with UV-B $\left(8 \mathrm{~W} \cdot \mathrm{m}^{-2}\right)$ for 5 min or covered with black paper (control) and left either in darkness or under constant white light $\left(100 \mu \mathrm{mol} \cdot \mathrm{m}^{-2} \cdot \mathrm{s}^{-1}\right)$ for the indicated time. Day 0 means $1 \mathrm{~h}$ after the treatment. Non-irradiated leaf halves served as a control. Pigments were separated by HPLC with detection by absorbance at $436 \mathrm{~nm}$ (chlorophylls) or $405 \mathrm{~nm}$ (carotenoids) and their content was determined from the area under the peak of the chromatogram using the extinction coefficients listed in Additional file 2: Table S2. Statistical significance of the differences between treatments was assessed with one-way ANOVA and the results of this analysis are listed in Additional file 3: Table S3

(UVL) samples, resulting in a statistically relevant difference between OUVL and 4UVL, as well as between 1UVL and 4UVL (Additional file 3: Table S3). Meanwhile, in control leaves (control continuous light-CL) the chlorophyll content remained stable or even slightly increased, resulting in a statistically significant difference of $p<0.005$ between 4UVL and 4CL for both chl a and chl b. The content of both chlorophylls in dark-adapted samples decreased both in treated (UV-B, then darknes$\mathrm{s}-\mathrm{UVD}$ ) and un-treated (control darkness-CD) ones, leading to a statistically significant difference of $p<0.005$ for $4 \mathrm{CD}$ vs $4 \mathrm{CL}$ and $4 \mathrm{UVD}$ vs $4 \mathrm{CL}$ for chl a and chl b, as well as lower but still statistically significant differences for the preceding days. However, the dynamic of these processes was different. While in UV-B treated leaves (UVD) the decrease was slow and steady from day 1 on, in the control (CD) it was pretty rapid after 3 days. UV-B treatment slowed down chlorophyll degradation. On day 4 in UVD samples chl a and chl b amounted to $129 \%$ and $153 \%$ of that observed in CD respectively. This difference was not statistically significant. However, whereas a difference between day 0 and day 4 was statistically significant for $\mathrm{CD}$, no statistical significance was observed for UVD. 4 days after irradiation the levels of both chlorophylls were clearly lower in UVL than in CL and similar to CD leaves. During treatment, the chl a/b ratio did not change significantly for CL samples, but increased in CD samples. In both UV-B treated samples this ratio was lower than in the corresponding controls (Fig. 4c). Differences of $p<0.005$ were noted between day 4 in UVL leaves and its CL control, as well as between 4 UVD and 4CD. Statistically significant differences were found already on 3rd day (i.e. $3 \mathrm{UVL}$ vs $3 \mathrm{CL}$, and $3 C D$ vs $3 U V D)$.

Similar trends were observed for all carotenoids tested (Fig. 4d-g). Again, in control samples kept in continuous light, the contents of violaxanthin, lutein and $\beta$-carotene increased or stayed unchanged. On the other hand, dark treatment led to a decrease in all carotenoids tested, what manifests as a statistically significant difference between $4 \mathrm{CD}$ and 1 to $4 \mathrm{CL}$. UV-B irradiation either did not influence the effect of darkness (violaxanthin, Fig. 4d) or slightly inhibited it (see: neoxanthin, lutein and $\beta$ carotene Fig. 4e-g), although the difference was not statistically significant. After a transient increase on day 1 , the decrease in carotenoid levels in UVL leaves on day 4th was either similar (lutein and violaxanthin), $50 \%$ lower (neoxanthin), or slightly higher ( $\beta$-carotene) than in the darkened control.

Bearing in mind the fact that the experimental treatment applied led to a decrease in the photosynthetic pigment content, we examined how these changes influenced photosynthetic performance. We assessed the yield of PSII via the measurement of chlorophyll fluorescence (Fig. 5a). The differences between maximum quantum yield of PSII (QYmax) levels were more clearly visible than these between levels of photosynthetic pigments. QYmax stayed unchanged in the control leaves kept in continuous light (no statistically significant differences between subsequent days in CL leaves). Leaves treated with UV-B prior to being transferred to continuous light showed a fast and very pronounced decrease in QYmax, consistent with the yellowing of the samples. These differences manifest as statistically significant between 3UVL and other leaves from this series (0UVL, 1UVL, 2UVL). In the CD leaves, the quantum yield decreased, first slowly, and from day 3 on, quite rapidly. Leaves treated with UV-B and darkened showed a steady decrease in QYmax, which resulted in higher values of this parameter on day 3 and 4 than in CD leaves (statistically significant difference with $p<0.005$ ), which corresponds to the slightly higher amounts of chlorophylls in those samples.

The changes in pigment contents were also accompanied by changes in protein levels. Quantitatively extracted total proteins were separated by SDS-page (Fig. 5b). The amount of proteins decreased in all but CL leaves. The loss of proteins in darkened samples was slower when they were UV-B pre-treated. The amount of D1 protein of PSII was also examined and showed similar trends to total proteins (Fig. 5b). Interestingly, a lower mass product resulting from UV-B-induced degradation could be observed in UV-B-treated samples. This product, present $1 \mathrm{~h}$ after irradiation (day 0 ), was no longer visible after 1 day in the light exposed sample. In darkness its degradation was very slow and the product was still clearly visible even after 4 days.

In order to see if the influence on photosynthetic processes was also reflected at the level of expression of photosynthesis-related genes, quantitative real-time PCR analysis was carried out (Fig. 5c and d). Typical, photosynthesis-related gene transcripts, RIBULOSE 


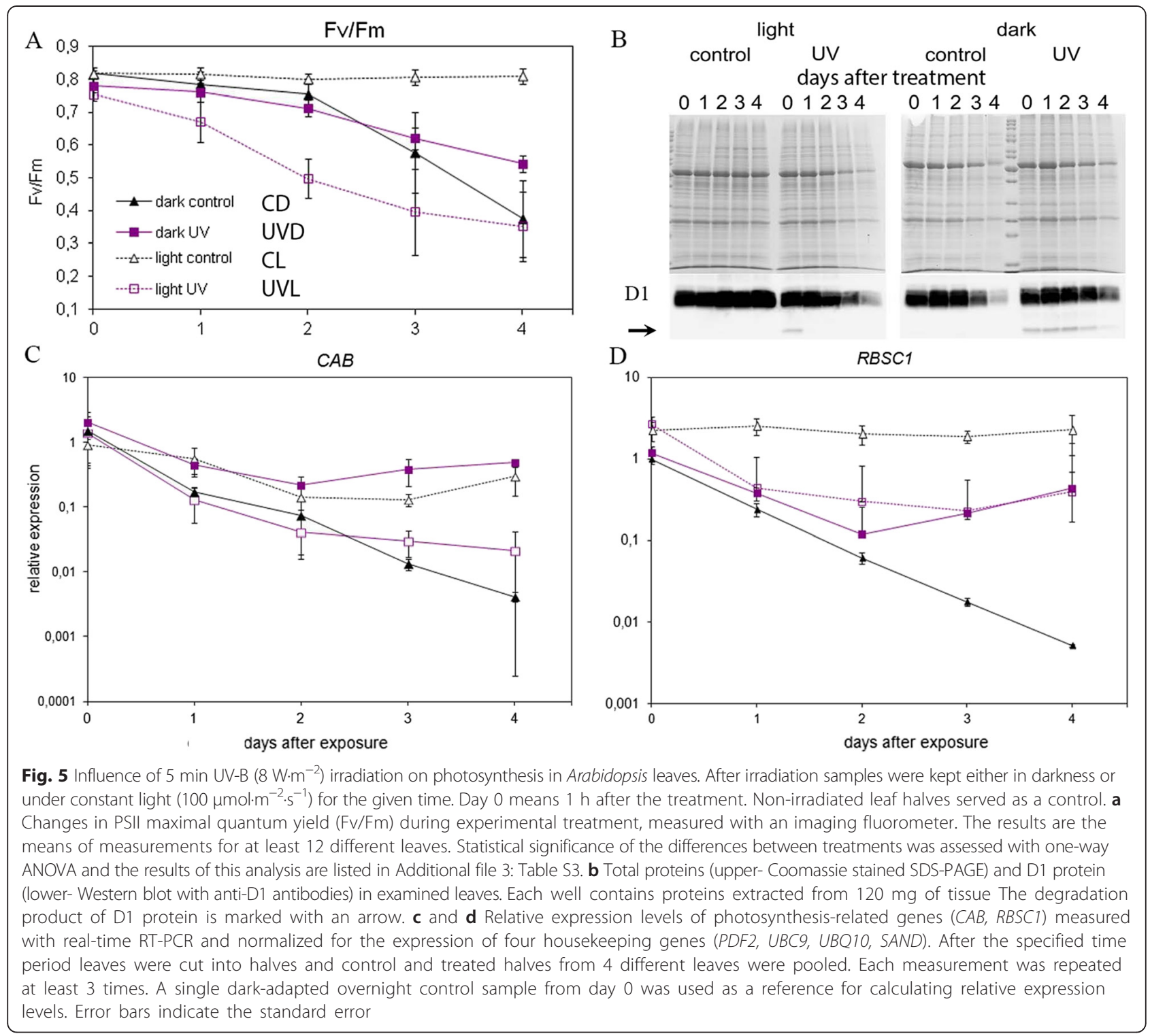

BISPHOSPHATE CARBOXYLASE SMALL CHAIN $1 A$ (RBCS1A) and CHLOROPHYLL A/B BINDING PROTEINS (CABs, including $C A B 1$ and $C A B 2$ ), were analyzed (Fig. $5 \mathrm{c}$ and d). Whereas the amount of RBCS1 mRNA stayed unchanged in the CL leaves even after 4 days, it decreased constantly in the darkened control leaves. On day 4 the transcript level of this gene was similar in UVD and UVL samples reaching a level almost 6 times lower than that observed in $\mathrm{CL}$ samples. The dark-induced decrease in control leaves was very rapid. After darkness exceeding 4 days (4 days plus overnight pre-treatment) the amount of RBCS1 reached only $0,3 \%$ of that observed in the leaves kept in continuous light.

The time-course of changes in the $C A B$ transcript level was slightly different (Fig. 5c). The steady-state level of this gene decreased during the experiment, with the most drastic drop in the darkened control samples. 4 days after treatment the amount of $C A B$ was similar in $\mathrm{CL}$ and UVD leaves. The decrease in UVL leaves was clearly faster, reaching only $0,13 \%$ of the transcript present on day 0. Finally, in darkened control leaves, at the end of the experiment, the $C A B$ transcript level was only $1,1 \%$ of that present in leaves kept in continuous light.

\section{Senescence and cell death}

As leaf yellowing and changes in photosynthetic efficiency often accompany senescence the level of senescenceassociated genes (SAGs) was also analyzed (Fig. 6). The first of these was $S A G 13$, an early senescence marker [12]. Interestingly, only $1 \mathrm{~h}$ after the treatment (day 0 ) the level 


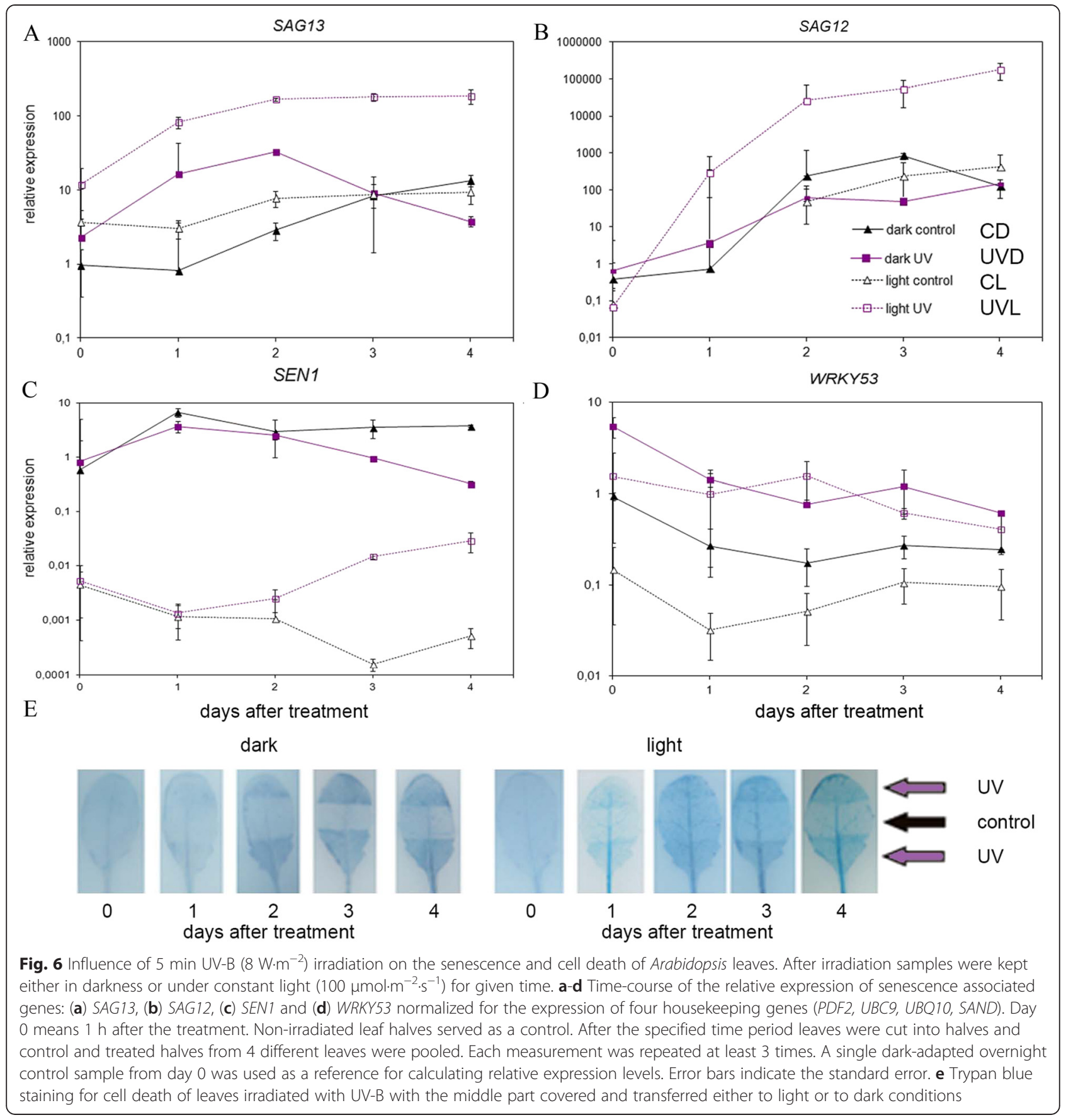

of this gene was slightly higher in irradiated samples as compared to control ones (Fig. 6a, compare UVD vs CD and UVL vs CL on day 0). The amounts of SAG13 transcripts increased very strongly in UVL leaves up to the second day, and stayed at the same elevated level on day 3 and 4. Finally, its level was almost 20 times higher than in CL leaves. As in CL, in darkened samples the amount of SAG13 started to increase between the 1st and 2nd day, but in CD leaves it continued to increase until day 4. An interesting situation was observed for UV irradiated and darkened samples. After strong up-regulation on days 1 and 2, the amount of SAG13 started to decline, reaching the level similar to CL samples on the 4th day. Nevertheless, this level was still higher as compared to nonirradiated darkened leaves.

The second gene tested was $S A G 12$, a late senescence marker [12] (Fig. 6b). The steady-state level of SAG12 transcript increased strongly in all samples tested in a time-dependent manner. Similarly to SAG13 the highest level of this gene transcript was observed in UV-B 
irradiated samples kept in continuous light. After 4 days the amount of this transcript increased by 442 times as compared with CL leaves. Interestingly, the changes in the SAG12 gene in all but UVL samples were similar, though with slightly different kinetics.

The expression of SEN1, another senescence marker was tested in addition to SAG12 and SAG13 [50]. Its expression depended mostly on light (Fig. 6c). It was very strongly induced in darkened samples starting from day 0 and lower in samples illuminated with continuous light. Prolonged night caused a very strong upregulation of SEN1, by 130 times as compared to leaves taken directly from the photoperiod $2 \mathrm{~h}$ after the light onset (day 0, compare CD and CL). Finally, on the 4th day the amount of SEN1 transcript was over 7.300 times higher in darkened leaves than in those kept in continuous light. The UV effect started to be visible between the 2nd and 3rd day after irradiation. At this time, the amount of SEN1 started to decrease in UVD leaves and to increase in UVL ones. 4 days after irradiation the level of this gene transcript was over 11 times higher in CD leaves than in UVD ones. The opposite effect was observed in samples from continuous light. In this case, the level of SEN1 transcript was 57 times higher in UVL leaves as compared to non-irradiated ones.

The steady-state level of WRKY53, a transcription factor up-regulated during early senescence, was also examined. Both darkness and UV-B treatment caused an increase in the level of this gene as compared to samples from constant light (Fig. 6d). Prolonged night caused over a 6-fold increase in the transcript level of this gene (compare CD and CL at the day 0). UV-B acted stronger than darkening, and the effect of UV-B and darkness was synergistic as the strongest, by over 39 times, upregulation was observed in UVD samples. The amount of WRKY53 changed over time, decreasing in all but the CL leaves. In control samples from constant light it transiently decreased 1 day after irradiation, but finally reached the same level as on day 0 . On the 4th day the highest level of WRKY53 was observed in both UV-B irradiated samples (6 times higher than in CL ones).

Finally, the occurrence of cell death in the leaves was studied using trypan blue staining (Fig. 6e). UV-B caused the gradual appearance of cell death irrespective of light conditions. Dark-treated leaf parts did not show trypan blue staining until day 4 and even then it was faint compared to that induced by UV-B.

\section{Anthocyanin content}

It is well known that anthocyanin synthesis is strongly up-regulated not only by visible light but also by UV-B. However, macroscopic observation of the samples treated under our experimental conditions did not confirm this up-regulation. Thus, we checked both the expression of genes involved in anthocyanin synthesis and the content of those pigments more carefully (Fig. 7a). Consistent with visual observations, a very strong increase in the levels of anthocyanins was observed in leaves transferred to continuous light. At the end of experiment, the anthocyanin content in leaves from constant light was 36 times higher than that observed in dark-treated leaves. Interestingly, treatment with UV-B almost completely abolished this response. The anthocyanin level was stable in darkened leaves independent of UV-B irradiation.

We also checked the expression of the genes involved in anthocyanin synthesis, PAL1 and CHS. The expression of PAL1 was very strongly down-regulated in darkened samples, whereas it stayed nearly unchanged in those undergoing constant illumination (Fig. $7 \mathrm{~b} ; \mathrm{CD}$ vs $\mathrm{CL}$ ). On the 4th day, the level of transcript was almost 200 times higher in leaves from constant light than in those from dark conditions. Interestingly, the effect of darkness was weaker in UV-B irradiated samples. Starting from the 1st day after irradiation the amount of PAL1 transcript was from 1.5 to over 5 times higher in UV-B treated samples than in dark controls.

A similar strong effect of darkness was observed for CHS (Fig. 7c). Prolonged night caused a decrease in the mRNA of this gene (day 0). Its level was 90 times higher in illuminated samples (CL) than in darkened ones independent of UV-B treatment (compare CD and UVD). The decrease in CHS level in control leaves left in darkness progressed during the experiment. On the 4th day this level was 123800 times higher in control leaves from constant light than in darkened ones. UV-B downregulated the CHS level in leaves from the light (3-8 times as compared to $\mathrm{CL}$ ). In dark-treated leaves UV-B up-regulated the amount of this transcript starting from the 2nd day after irradiation.

\section{Macroscopic appearance of leaves of selected mutants}

In order to elucidate possible mechanism(s) underlying the observed UV-B effect, two mutants were examined: uvr8-6, depleted of UV-B receptor [39] and $m c p 2 d$, lacking metacaspase $2 \mathrm{~d}$ involved in programmed cell death [40].

The former one was used to check the involvement of UVR8-activated signalling pathway in either inhibition or promotion of chlorophyll degradation in darkness and in light respectively. The $m c p 2 d$ mutant served to test the possible role of this metacaspase in chlorophyll degradation in Arabidopsis leaves illuminated after irradiation.

The dark-induced leaf yellowing was slowed down in $m c p 2 d$ leaves as compared with WT ones (Additional file 4: Figure S1, dark control). Leaves of $u v r 8$ plants were more sensitive to UV-B-induced damage. The damage symptoms 

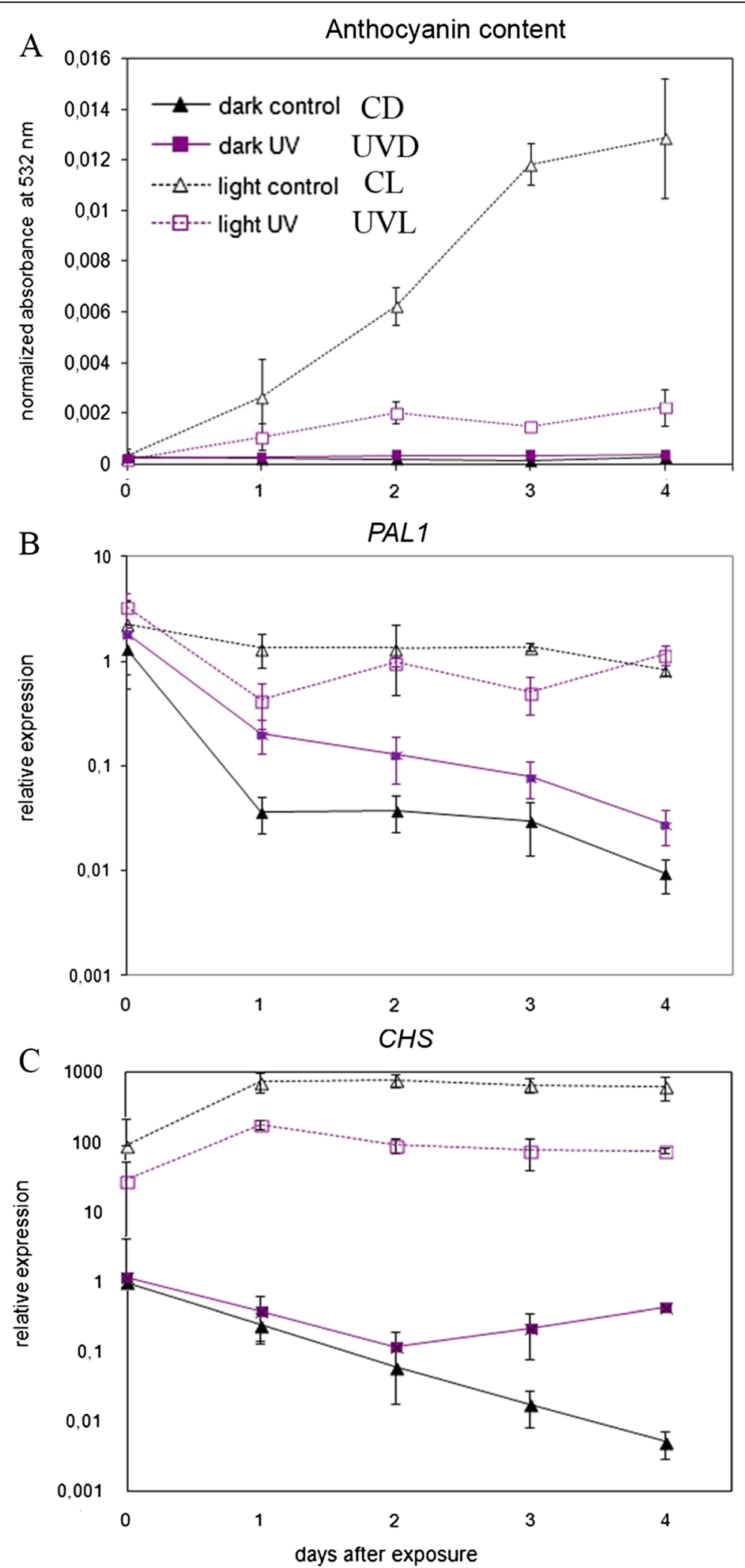

Fig. 7 (See legend on next page.) 
(See figure on previous page.)

Fig. 7 Influence of UV-B on anthocyanins in Arabidopsis leaves. After the specified time period leaves were cut into halves and control and treated halves from 4 different leaves were pooled. Each measurement was repeated at least 3 times. Error bars indicate the standard error. a Anthocyanin content was analyzed by measuring absorbance at $532 \mathrm{~nm}$ and normalized to fresh weight in examined leaves. b and $\mathbf{c}$ Time-course of the relative expression of genes involved in anthocyanin biosynthesis: (b) PAL1 and (c) CHS normalized for the expression of four housekeeping genes (PDF2, UBC9, UBQ10, SAND). A single dark-adapted overnight control sample from day 0 was used as a reference for calculating relative expression levels

were more severe in $u v r 8$ leaves darkened after irradiation. The influence of UV-B on chlorophyll degradation was comparable in WT Col and uvr8 and mcp2d mutants independent on the light conditions (Additional file 4: Figure S1).

\section{Discussion}

Treatment with high doses of UV-B alleviates darkeninginduced senescence symptoms in detached Arabidopsis leaves

One of the most evident symptoms of senescence is yellowing which originates from faster catabolism of chlorophylls in comparison to yellow pigments. This process may be a result of natural senescence or stress (biotic or abiotic). Senescence is also induced in leaves that are detached and stored in darkness or individually darkened on a plant kept in a photoperiod [51]. Yellowing may be delayed by interference with the chlorophyll degradation pathway, as observed in numerous mutants, collectively known as "stay-green". Those mutants can be classified as either functional, i.e. those with delayed overall senescence, or cosmetic, i.e. those where only chlorophyll degradation is delayed while other aspects of senescence progress normally [52].

In our model, the darkening of detached leaf halves resulted in a decrease in both chl a and chl b content and an increase in the chl a/chl b ratio. These trends are similar to those observed before [5, 53]. However, we did not observe such a significant amount of chlorophyll degradation products as previously reported [53]. Some amount of pheophytins was found only after 4 days of darkness (not shown).

A much higher retention of carotenoids is typical of the senescing leaves of almost all plants. Usually, the carotenoid/chlorophyll ratio increases [54]. Changes in the levels of neoxanthin, violaxanthin, lutein and $\beta$-carotene occur in parallel $[55,56]$. Our results are consistent with those observations. The level of carotenoids did not change during the first two days of darkening, and started to decrease from the 3rd day. Taken together, these results indicate that our system is an appropriate experimental model of senescence.

Sub-lethal doses of UV-C, UV-B and gamma irradiation are widely used in post-harvest technology. Beneficial, hormetic effects of such irradiation include the sanitization of fresh vegetables and fruits, an increase in phenolic compounds, in the content of lycopene and other pigments, the up-regulation of antioxidant content and antioxidant enzyme activity (for a rewiev see: [57]). Treatment with ultraviolet light may also influence chlorophyll content. Irradiation with a relatively high dose of UV-B $\left(8.8 \mathrm{~kJ} \cdot \mathrm{m}^{-2} 2\right)$ has been shown to delay the yellowing of broccoli florets ([37]) and lime peel during storage [38]. This yellowing results from the storage of detached plant parts in darkness. It has been shown that dark storage induces senescence and the expression of senescence-associated genes in broccoli starting after 3 days of storage [58].

We show that a similar dose-dependent effect can be observed in detached Arabidopsis leaves kept in darkness after irradiation with $8 \mathrm{~W} \cdot \mathrm{m}^{-2} \mathrm{UV}-\mathrm{B}$ (i.e. $2,4 \mathrm{~kJ} \cdot \mathrm{m}^{-2}$ ) (Fig. 2). Our results indicate that high-doses of UV-B interfere with chlorophyll degradation in darkness and slow it down. The contents of all photosynthetic pigments tested, except neoxanthin and violaxanthin, were slightly lower in irradiated leaves during the first 2 days after treatment (Fig. 4). However, starting from day 3 chlorophyll degradation in control leaves progressed much faster and the balance reversed in favor of UV-B treated plants. The same was observed for photosynthesis efficiency (Fig. 5a). The maximal quantum yield of PSII was higher in UV treated leaves than in control (only darkened) ones. Thus, starting from 3 days after irradiation the positive effects of the treatment outweighed the negative ones. Chlorophyll $\mathrm{a}$ and $\mathrm{b}$ were degraded in darkened samples but the degradation rate of both pigments was modulated by UV irradiation. The chl a/chl $\mathrm{b}$ ratio increased in darkened control leaves (Fig. 4b), in accordance with previous reports [53]. This probably results from the faster degradation of LHCII-derived (Light-harvesting complex II) chlorophylls mediated by a complex of STAY-GREEN1 (SGR1) with chlorophyll catabolic enzymes [59], as it is counteracted in a mutant with impaired SGR1 protein function (nonyellowing1-1, nye1-1) during mild stress treatment [60]. Interestingly, in UV-treated samples the $\mathrm{chl} \mathrm{a} / \mathrm{chl} \mathrm{b}$ ratio remained almost unchanged during the whole time-course of the experiment. This suggests that in the absence of visible light UV-B activated signals can interfere with a specific pathway of LHCII degradation. In our system the Lhcb1 protein level remained unchanged during the experiment (Western blot data not shown). It has been observed that the degradation of Lhc proteins starts later than degradation of D1 protein [60]. It is likely 
that the components of LHCII, namely proteins and pigments, are degraded sequentially and that chlorophyll degradation precedes other processes.

The amounts of $\beta$-carotene and lutein were slightly higher after UV irradiation (Fig. $4 \mathrm{f}$ and g). These pigments are constituents of the photosynthetic protein pigment complexes, PSII and LHCII respectively. The increased retention of $\beta$-carotene after UV-B treatment correlates with the slower degradation of D1 protein and may result from the increased stability of the whole complex.

Lutein and other xanthophylls which build LHCII complexes are important in photoprotection mechanisms $[61,62]$. The higher level of lutein and unchanged level of violaxanthin after UV-B pretreatment may result from a slower degradation of LHCII. Neoxanthin was the only pigment that showed an immediate decrease after UV-B treatment. It is known that neoxanthin absorbs UV and that it can photoisomerize upon excitation [63]. Although other carotenoids also can photoisomerize, the effect for neoxanthin is the strongest and can reach $10 \%$ of this pigment's content. Thus, the observed decrease may result from photoisomerization. Light is necessary for an effective conversion of neoxanthin isomers to the primary conformation. Since there is no light in our system, the process is very slow and the level of neoxanthin in UV treated samples remains lowest during the whole time-course of the experiment.

All UV effects on photosynthesis in darkened leaves were accompanied with a slower decrease in total protein level, a slower decrease in the amount of D1 protein as well as a slower decrease in the level of photosynthesisrelated transcripts (Fig. 5). This is consistent with observed changes in photosynthetic efficiency. The specific product of UV-induced D1 protein cleavage was present after the treatment (Fig. 5b, day 0). As in [35], we showed the persistence of this product in the absence of light, in our case for up to 4 days. Taken together, our results suggest that UV-B treatment alleviates the effects of darkening on the functionality of the photosynthetic apparatus.

The examination of expression levels of senescenceassociated genes showed that the UV effect observed was not caused by a simple retardation of senescence (Fig. 6). On the one hand, the mRNA levels of SAG12 and SEN1 were lower after short UV-B treatment in samples darkened for 3 days, when the differences in chlorophyll degradation started to be visible. On the other hand, over the whole experiment the expression of WRKY53 was elevated in irradiated samples. The amount of SAG13 transcripts was higher for the first 2 days after the treatment and started to decrease on 3rd day. This rather complicated pattern of senescenceassociated gene regulation suggests that UV-B interferes with their expression; it does not supplement darkeninginduced senescence but tends to modulate it.
It should be noted that the advantageous effects of UV started to be visible at the same time as cell death symptoms appeared, confirmed by trypan blue staining. This shows that in our experimental system leaves are highly susceptible to both the damaging effects of UV-B and to the beneficial ones. The exact mechanism of the inhibition of dark-induced leaf yellowing by high doses of UV remains to be determined. To date, studies on the UV-dependent inhibition of chlorophyll degradation have been performed using broccoli florets and lime peel $[37,38]$. It has been shown that UV-B inhibits the activity of chlorophyll peroxidase [37] and recently, the specifically affected by UV-B chlorophyll peroxidase $\mathrm{C}$ has been identified [64].

Our results demonstrated that the inhibition of chlorophyll degradation in darkness was independent of the UV-B photoreceptor, UVR8, since leaves of the $u v r 8$ mutant showed the same symptoms as those of WT plants i.e. slowed dark-induced chlorophyll degradation (Additional file 4: Figure S1, dark). The more severe damage observed in $u v r 8$ mutants probably results from the lack of UVR8-regulated protective mechanisms It is in line with the hypothesis that UVR8 mediates responses to low UV-B doses, whereas high doses of UV-B activate an independent pathway involving the mitogenactivated protein kinase (MAPK) cascade [3]. This cascade regulates UV-B dependent programmed cell death (PCD) in plants. One of the regulators of cell death induced during biotic and abiotic stresses in Arabidopsis is metacaspase 2d (MCP2d). Experiments employing the $m c p 2 d$ mutant demonstrated that the effects of UV-B observed in this mutant were comparable to the wild type (Additional file 4: Figure S1, dark). Thus, the inhibition of dark-induced chlorophyll degradation was not due to the inhibition of the activity of this metacaspase.

\section{Visible light influences UV-B action}

Visible light dramatically affected the response of leaves to high doses of UV. While darkened leaves stayed green even 4 days after UV irradiation, yellowing was observed in leaves transferred to continuous light starting from the 2nd day (Fig. 3). This yellowing was a result of a decrease in the levels of all photosynthetic pigments and was accompanied by a decrease in photosynthesis efficiency (Figs. 3 and 5). In contrast to the senescing CD samples, in UVL ones the $\mathrm{chl} \mathrm{a} / \mathrm{chl} \mathrm{b}$ ratio decreased. The decrease resembled the effect observed in the nye11 mutant during mild salt stress (Sakuraba et al. 2014). This suggests that the level of activation of different pigment degradation pathways during UV-mediated senescence in light was not the same as during darkinduced senescence. In particular, the specific, SGR1dependent pathway of LHCII degradation appeared not to be activated. 
The cumulative dose of UV-B necessary to decrease the chlorophyll level has been calculated for Pisum sativum as $300 \mathrm{~kJ} \cdot \mathrm{m}^{-2}$ [65]. In Arabidopsis grown in a $12 \mathrm{~h}$ light photoperiod with photosynthetic photon flux density (PPFD) of $300 \mu \mathrm{mol} \cdot \mathrm{m}^{-2} \cdot \mathrm{s}^{-1}$ plus $6 \mathrm{~kJ} \cdot \mathrm{m}^{-2} \cdot \mathrm{d}^{-1}$ of UV-B [34], the chlorophyll content increased [34]. Even the addition $2,4 \mathrm{~W} \cdot \mathrm{m}^{-2}$ of UV-B for $5 \mathrm{~h}$ (i.e. $8,64 \mathrm{~kJ} \cdot \mathrm{m}^{-2}$ dose) to $40 \mu \mathrm{mol} \cdot \mathrm{m}^{-2} \cdot \mathrm{s}^{-1}$ of PPFD did not influence the chlorophyll level in 29 day old Arabidopsis [66]. In this experiment the content of both chlorophylls, lutein, violaxanthin and antheraxanthin remained unchanged as long as 4 days after UV-B irradiation. The UV-B dose used in our experiment e.g. $2,4 \mathrm{~kJ} \cdot \mathrm{m}^{-2}$ was comparable with above experiments. The main differences were: using detached leaves from plants grown in a shorter (10 h of light) photoperiod and using constant illumination after the treatment. However, when leaves after UV-B treatment were transferred back to the photoperiod, the same symptoms were observed, but with slower kinetics (data not shown). The influence of PAR intensity during Arabidopsis growth on the effect of UV-B has been shown before $[67,68]$. The light intensity during the growth was lower in our experiment $\left(70 \mu \mathrm{mol} \cdot \mathrm{m}^{-2} \cdot \mathrm{s}^{-1}\right)$ than those reported by [66] (130 $\left.\mu \mathrm{mol} \cdot \mathrm{m}^{-2} \cdot \mathrm{s}^{-1}\right)$ and [34] $\left(300 \mu \mathrm{mol} \cdot \mathrm{m}^{-2} \cdot \mathrm{s}^{-1}\right)$. Thus, it is possible that either light intensity and/or the duration of the photoperiod modulate the UV-B effects on photosynthesis (compare [28]). Additionally, Götz et al. [68] showed that a low level of UV-B during growth leads to a low accumulation of protective isoflavonoids. This may lead to a higher susceptibility of the plants to UV-B irradiation, compared to the plants grown in the presence of higher UV-B levels. While in our growth chamber UV-B irradiation was completely excluded, no data on the intensity of UV-B during plant growth are provided either by Moon's or Poulson's groups [34, 66].

The expression of senescence-associated genes has been shown to be up-regulated in Arabidopsis grown under white light supplemented with UV-B [13]. Similarly, in our system the expression of all senescenceassociated genes tested was strongly up-regulated in UVL leaves (Fig. 6). A comparison of the results obtained for darkened and illuminated samples shows that PAR is a key factor in the induction of senescenceassociated genes by UV-B. In consequence, the observed decrease in photosynthesis resulting from chlorophyll degradation seems to be a result of the initiation of the senescence process.

Interestingly, UV-B pretreatment completely inhibited the accumulation of anthocyanins in continuous light. The expression of anthocyanin regulatory genes as well as anthocyanin accumulation have been shown to be strongly increased by UV-A and by visible light, mainly in the blue range. UV-B alone is much less effective, but it acts synergistically with visible light $[29,69,70]$. In our experiments the expression levels of PAL1 and CHS were elevated in leaves continuously illuminated with white light as compared to darkened samples (Fig. 7). UV-B either did not influence $(C H S)$ or slightly reduced (PAL1) this increase. In contrast, while anthocyanin accumulation was very strongly enhanced by white light, UV-B pretreatment counteracted this effect. The activation of the senescence program by UV-B irradiation, might eliminate the need for the production of photoprotective pigments.

Although the impact of UV-B on photosynthesis, chlorophyll degradation, expression of senescenceassociated genes was modulated by the subsequent light conditions, cell death was observed in samples both darkened and illuminated with continuous light. Visible light proved to be necessary for the proper course of a senescence program triggered by a high dose of UV-B. Light is required for the onset of cell death under nutrient-limiting conditions [71] and in Arabidopsis protoplasts after UV-C treatment [72]. Chloroplast delivered signals, most probably ones connected with the production of reactive oxygen species, seem to be involved in executing senescence and cell death [73-75]. Indeed, in our system the senescence program leading to cell death was initiated in illuminated samples. In darkened ones other processes leading to cell death were activated. The signaling pathways leading to cell death after UV-B irradiation and darkening do not act synergistically but seem to be mutually exclusive to some extent.

Again, UVR8-dependent signalling pathway was not involved in UV-B-induced chlorophyll degradation in light (Additional file 4: Figure S1).

\section{Conclusions}

Our results show the importance of the light conditions applied after the irradiation with high dose of UV-B. These conditions influenced the expression of photosynthesisrelated and senescence-associated genes, chlorophyll degradation and photosynthesic efficiency. Short UV-B treatment promoted leaf yellowing in light and inhibited it upon the storage of leaves in darkness. However, irrespective of light conditions, visible cell death symptoms appeared 3 days after UV-B irradiation.

\section{Additional files}

Additional file 1: Table S1. Sequences of primers used in this study. (DOCX $15 \mathrm{~kb}$ )

Additional file 2: Table S2. Extinction coefficients in HPLC solvent. (DOCX $10 \mathrm{~kb}$ )

Additional file 3: Table S3. Analysis of statistical relevance in changes of photosynthetic pigments level (a: chlorophyll a, b: chlorophyll b, c: chlorophyll a/chlorophyll b ratio, d: violaxanthin, e: neoxanthin, f: lutein, g: $\beta$-carotene) as well as in yield of Photosystem II (h). (PDF 325 kb) 
Additional file 4: Figure S1. Photographs of the detached leaves of 6-week old A. thaliana WT, mcp2d and uvr8 mutants with one half covered with black paper, and another half irradiated with UV-B $\left(8 \mathrm{~W} \cdot \mathrm{m}^{-2}\right)$ for $5 \mathrm{~min}$ and (A) left in darkness or (B) illuminated with white light $\left(100 \cdot \mu \mathrm{mol} \cdot \mathrm{m}^{-2} \cdot \mathrm{s}^{-1}\right)$ for 4 days. (TIF $10951 \mathrm{~kb}$ )

\section{Abbreviations}

CAB: CHLOROPHYLL AVB BINDING PROTEIN; CD: Control dark, non-treated leaves dark adapted and left in the darkness; chl: Chlorophyll; CHS: CHALCONE SYNTHASE; CL: Control light, non-treated leaves left under continuous light (100 $\mu \mathrm{mol} \cdot \mathrm{m}^{\text {s-2-1 }}$ ); LHC II: Light-harvesting complex II; mcp2d: metacaspase 2d; nye1-1: non-yellowing 1-1; PAL 1: PHENYLALANINE AMMONIA-LYASE 1; PAR: Photosynthetically active radiation; PPFD: Photosynthetic photon flux density; PSII: Photosystem II; QYmax: Maximum quantum yield of Photosystem II; RBCSIA: RIBULOSE BISPHOSPHATE CARBOXYLASE SMALL CHAIN 1A; ROS: Reactive oxygen species; SAG: SENESCENCE ASSOCIATED GENE; SEN1: SENESCENCE1; SGR1: STAY-GREEN1; UV: Ultraviolet; UVD: UV dark—dark adapted leaves irradiated for $5 \mathrm{~min}$ with $8 \mathrm{~W} \cdot \mathrm{m}^{-2}$ of UV-B and left in darkness; UVL: UV light-leaves irradiated for 5 min with $8 \mathrm{~W} \cdot \mathrm{m}^{-2}$ of UV-B and left under continuous light (100 $\left.\mu \mathrm{mol} \cdot \mathrm{m}^{-2} \cdot \mathrm{s}^{-1}\right)$; uvr8: uvb-resistance 8.

\section{Competing interests}

The authors declare that they have no competing interests.

\section{Authors' contributions}

OS: participated in the design of the study, conducted the experiments, discussed and analyzed the data and improved the manuscript. JG: participated in the HPLC analysis, interpreted the results and improved the manuscript. AKB: conceived of the study, participated in its design and coordination, drafted the manuscript. HG: participated in the design of the study and in the discussion of results. All authors read and approved the final manuscript.

\section{Acknowledgements}

The study was supported by the Polish National Science Centre, grant no. UMO-2011/03/D/NZ3/00210. The Faculty of Biochemistry, Biophysics and Biotechnology of Jagiellonian University is the beneficiary of structural funds from the European Union; grant no. POIG.02.01.00-12-064/08. The Faculty of Biochemistry, Biophysics and Biotechnology is a partner of the Leading National Research Center (KNOW) supported by the Ministry of Science and Higher Education. HPLC measurements were performed in NanoFun Laboratories, POIG.02.02.00-00-025/09.

\section{Author details}

'Department of Plant Biotechnology, Faculty of Biochemistry, Biophysics and Biotechnology, Jagiellonian University, Gronostajowa 7, Krakow 30-387, Poland. ${ }^{2}$ Current address: Institute of Biochemistry and Biophysics, Polish Academy of Sciences, Warszawa 02-106, Poland. ${ }^{3}$ Laboratory of Biological Physics, Institute of Physics, Polish Academy of Sciences, Al. Lotników 32/46, Warszawa 02-668, Poland. ${ }^{4}$ The Malopolska Centre of Biotechnology, Jagiellonian University, Gronostajowa 7, Krakow 30-387, Poland.

\section{Received: 10 August 2015 Accepted: 17 November 2015} Published online: 25 November 2015

\section{References}

1. Hollósy F. Effects of ultraviolet radiation on plant cells. Micron. 2002;33:179-97.

2. Brown BA, Jenkins GI. UV-B signaling pathways with different fluence-rate response profiles are distinguished in mature Arabidopsis leaf tissue by requirement for UVR8, HY5, and HYH. Plant Physiol. 2008;146:576-88.

3. Nawkar GM, Maibam P, Park JH, Sahi VP, Lee SY, Kang CH. UV-induced cell death in plants. Int J Mol Sci. 2013;14:1608-28.

4. Teramura AH. Effects of ultraviolet-B radiation on the growth and yield of crop plants. Physiol Plant. 1983;58:415-27.

5. Frohnmeyer $\mathrm{H}$, Staiger D. Ultraviolet-B radiation-mediated responses in plants. Balancing damage and protection. Plant Physiol. 2003;133:1420-8.

6. Sarvikas $P$, Hakala M, Pätsikkä E, Tyystjärvi T, Tyystjärvi E. Action spectrum of photoinhibition in leaves of wild type and npq1-2 and npq4-1 mutants of Arabidopsis thaliana. Plant Cell Physiol. 2006;47:391-400.
7. Boyko A, Greer M, Kovalchuk I. Acute exposure to UVB has a more profound effect on plant genome stability than chronic exposure. Mut Res Fund Mol Mech Mutagen. 2006;602:100-9.

8. Lake JA, Field KJ, Davey MP, Beerling DJ, Lomax BH. Metabolomic and physiological responses reveal multi-phasic acclimation of Arabidopsis thaliana to chronic UV radiation. Plant Cell Env. 2009;32:1377-89.

9. Pourtau N, Marès M, Purdy S, Quentin N, Ruël A, Wingler A. Interactions of abscisic acid and sugar signalling in the regulation of leaf senescence. Planta. 2004;219:765-72.

10. Munné-Bosch S, Alegre L. Die and let live: Leaf senescence contributes to plant survival under drought stress. Funct Plant Biol. 2004;31:203-16.

11. Diaz C, Saliba-Colombani V, Loudet O, Belluomo P, Moreau L, Daniel-Vedele F, et al. Leaf yellowing and anthocyanin accumulation are two genetically independent strategies in response to nitrogen limitation in Arabidopsis thaliana. Plant Cell Physiol. 2006;47:74-83.

12. Weaver LM, Gan S, Quirino B, Amasino RM. A comparison of the expression patterns of several senescence-associated genes in response to stress and hormone treatment. Plant Mol Biol. 1998;37:455-69.

13. John C, Morris K, Jordan B, Thomas B, A-H-Mackerness S. Ultraviolet-B exposure leads to up-regulation of senescence-associated genes in Arabidopsis thaliana. J Exp Bot. 2001;52:1367-73.

14. Kusano M, Tohge T, Fukushima A, Kobayashi M, Hayashi N, Otsuki H, et al. Metabolomics reveals comprehensive reprogramming involving two independent metabolic responses of Arabidopsis to UV-B light. Plant J. 2011;67:354-69.

15. Dunning CA, Chalker-Scott L, Scott JD. Exposure to ultraviolet-B radiation increases cold hardiness in Rhododendron. Physiol Plant. 1994;92:516-20.

16. Kakani VG, Reddy KR, Zhao D, Gao W. Senescence and hyperspectral reflectance of cotton leaves exposed to ultraviolet-B radiation and carbon dioxide. Physiol Plant. 2004;121:250-7.

17. Pradhan M, Nayak L, Joshi P, Mohapatra P, Patro L, Biswal B, et al. Developmental phase-dependent photosynthetic responses to ultraviolet- $B$ radiation: Damage, defence, and adaptation of primary leaves of wheat seedlings. Photosynthetica. 2008;46:370-7.

18. Kakani V, Reddy K, Zhao D, Sailaja K. Field crop responses to ultraviolet-B radiation: A review. Agric Forest Meteorol. 2003;120:191-218.

19. Hunt J, Mcneil D. Nitrogen status affects UV-B sensitivity of cucumber. Funct Plant Biol. 1998;25:79-86.

20. Jenkins ME, Suzuki TC, Mount DW. Evidence that heat and ultraviolet radiation activate a common stress-response program in plants that is alterd in the uvh6 mutant of Arabidopsis thaliana. Plant Physiol. 1997;115:1351-8.

21. Fedina IS, Grigorova ID, Georgieva KM. Response of barley seedlings to UV-B radiation as affected by $\mathrm{NaCl}$. J Plant Physiol. 2003;160:205-8.

22. Chalker-Scott L, Scott JD. Elevated ultraviolet-B radiation induces crossprotection to cold in leaves of rhododendron under field conditions. Photochem Photobiol. 2004;79:199-204.

23. Teklemariam T, Blake TJ. Effects of UVB preconditioning on heat tolerance of cucumber (Cucumis sativus L.). Env Exp Bot. 2003;50:169-82.

24. Bandurska H, Niedziela J, Chadzinikolau T. Separate and combined responses to water deficit and UV-B radiation. Plant Sci. 2013;213:98-105.

25. Paul ND, Moore JP, Mcpherson M, Lambourne C, Croft P, Heaton JC, et al. Ecological responses to UV radiation: Interactions between the biological effects of UV on plants and on associated organisms. Physiol Plant. 2012;145:565-81.

26. Wang H, Hao J, Chen $X$, Hao Z, Wang X, Lou Y, et al. Overexpression of rice WRKY89 enhances ultraviolet $B$ tolerance and disease resistance in rice plants. Plant Mol Biol. 2007:65:799-815.

27. Krizek DT. Influence of PAR and UV-A in determining plant sensitivity and photomorphogenic responses to UV-B radiation. Photochem Photobiol. 2004;79:307-15.

28. Kimura M, Yamamoto $Y$ Y, Seki M, Sakurai T, Sato M, Abe T, et al. Identification of Arabidopsis genes regulated by high light-stress using cDNA microarray. Photochem Photobiol. 2003;77:226-33.

29. Bieza K, Lois R. An Arabidopsis mutant tolerant to lethal ultraviolet-B levels shows constitutively elevated accumulation of flavonoids and other phenolics. Plant Physiol. 2001;126:1105-15.

30. Gao Q, Zhang L. Ultraviolet-B-induced oxidative stress and antioxidant defense system responses in ascorbate-deficient vtc1 mutants of Arabidopsis thaliana. J Plant Physiol. 2008;165:138-48.

31. Cen Y-P, Bornman JF. The response of bean plants to UV-B radiation under different irradiances of background visible light. J Exp Bot. 1990;41:1489-95. 
32. Deckmyn G, Martens C, Impens I. The importance of the ratio UV-B/ photosynthetic active radiation (PAR) during leaf development as determining factor of plant sensitivity to increased UV-B irradiance: Effects on growth, gas exchange and pigmentation of bean plants (Phaseolus vulgaris cv. Label). Plant Cell Env. 1994;17:295-301.

33. Hectors K, Prinsen E, De Coen W, Jansen MA, Guisez Y. Arabidopsis thaliana plants acclimated to low dose rates of ultraviolet $B$ radiation show specific changes in morphology and gene expression in the absence of stress symptoms. New Phytol. 2007;175:255-70.

34. Poulson ME, Boeger MRT, Donahue RA. Response of photosynthesis to high light and drought for Arabidopsis thaliana grown under a UV-B enhanced light regime. Photosynt Res. 2006;90:79-90.

35. Bergo E, Segalla A, Giacometti GM, Tarantino D, Soave C, Andreucci F, et al. Role of visible light in the recovery of photosystem II structure and function from ultraviolet-B stress in higher plants. J Exp Bot. 2003;54:1665-73.

36. Bolink EM, Van Schalkwijk I, Posthumus F, Van Hasselt PR. Growth under UV-B radiation increases tolerance to high-light stress in pea and bean plants. Plant Ecol. 2001;154:147-56.

37. Aiamla-Or S, Kaewsuksaeng S, Shigyo M, Yamauchi N. Impact of UV-B irradiation on chlorophyll degradation and chlorophyll-degrading enzyme activities in stored broccoli (Brassica oleracea L. Italica Group) florets. Food Chem. 2010;120:645-51.

38. Srilaong V, Aiamla-Or S, Soontornwat A, Shigyo M, Yamauchi N. UV-B irradiation retards chlorophyll degradation in lime (Citrus latifolia Tan.) fruit. Postharvestal Biol Technol. 2011;59:110-2.

39. Favory JJ, Stec A, Gruber H, Rizzini L, Oravecz A, Funk M, et al. Interaction of COP1 and UVR8 regulates UV-B-induced photomorphogenesis and stress acclimation in Arabidopsis. EMBO J. 2009;28:591-601.

40. Watanabe $\mathrm{N}$, Lam E. Arabidopsis metacaspase $2 \mathrm{~d}$ is a positive mediator of cell death induced during biotic and abiotic stresses. Plant J. 2011;66:969-82.

41. Alonso JM, Stepanova AN, Leisse TJ, Kim CJ, Chen H, Shinn P, et al. Genome-wide insertional mutagenesis of Arabidopsis thaliana. Science. 2003;301:653-7.

42. Van Leeuwe M, Villerius L, Roggeveld J, Visser R, Stefels J. An optimized method for automated analysis of algal pigments by HPLC. Marin Chem. 2006;102:267-75.

43. Inskeep WP, Bloom PR. Extinction coefficients of chlorophyll a and b in N, N-dimethylformamide and 80 \% acetone. Plant Physiol. 1985;77:483-5.

44. Karrer P, Jucker E. Carotenoids. New York: Elsevier; 1950

45. Mallams A, Waight E, Weedon B, Chapman DJ, Haxo F, Goodwin T et al. A new class of carotenoids. Chem Commun. 1967:6:301-2.

46. Craft NE, Soares JH. Relative solubility, stability, and absorptivity of lutein and. beta.-carotene in organic solvents. J Agricult Food Chem. 1992;40:431-4.

47. Łabuz J, Sztatelman O, Banaś AK, Gabryś H. The expression of phototropins in Arabidopsis leaves: Developmental and light regulation. J Exp Bot. 2012; 63:1763-71.

48. Vandesompele J, De Preter K, Pattyn F, Poppe B, Van Roy N, De Paepe A et al. Accurate normalization of real-time quantitative RT-PCR data by geometric averaging of multiple internal control genes. Genome Biol. 2002; 3:research0034.

49. Laemmli UK. Cleavage of structural proteins during the assembly of the head of bacteriophage T4. Nature. 1970;227:680-5.

50. Oh SA, Lee SY, Chung IK, Lee C-H, Nam HG. A senescence-associated gene of Arabidopsis thaliana is distinctively regulated during natural and artificially induced leaf senescence. Plant Mol Biol. 1996;30:739-54.

51. Weaver LM, Amasino RM. Senescence is induced in individually darkened Arabidopsis leaves, but inhibited in whole darkened plants. Plant Physiol. 2001;127:876-86.

52. Grassl J, Pružlnská A, HöRtensteiner S, Taylor NL, Millar AH. Early events in plastid protein degradation in stay-green Arabidopsis reveal differential regulation beyond the retention of $\mathrm{LHCl}$ and chlorophyll. J Proteom Res. 2012;11:5443-52

53. Pružinská A, Tanner G, Aubry S, Anders I, Moser S, Müller T, et al. Chlorophyll breakdown in senescent Arabidopsis leaves. Characterization of chlorophyll catabolites and of chlorophyll catabolic enzymes involved in the degreening reaction. Plant Physiol. 2005;139:52-63.

54. Lu C, Lu Q, Zhang J, Kuang T. Characterization of photosynthetic pigment composition, photosystem II photochemistry and thermal energy dissipation during leaf senescence of wheat plants grown in the field. J Exp Bot. 2001;52:1805-10.
55. Whitfield DM, Rowan KS. Changes in the chlorophylls and carotenoids of leaves of Nicotiana tabacum during senescence. Phytochemistry. 1974;13:77-83.

56. Young AJ, Wellings R, Britton G. The fate of chloroplast pigments during senescence of primary leaves of Hordeum vulgare and Avena sativum. J Plant Physiol. 1991;137:701-5.

57. Ribeiro C, Alvarenga N. Prospects of UV radiation for application in postharvest technology. Emir. J. Food Agric. 2012;24(6):586-597.

58. Page T, Griffiths G, Buchanan-Wollaston V. Molecular and biochemical characterization of postharvest senescence in broccoli. Plant Physiol. 2001; 125:718-27.

59. Sakuraba $Y$, Schelbert $S$, Park S-Y, Han S-H, Lee B-D, Andrès CB, et al. STAY-GREEN and chlorophyll catabolic enzymes interact at light-harvesting complex II for chlorophyll detoxification during leaf senescence in Arabidopsis. Plant Cell. 2012;24:507-18.

60. Sakuraba Y, Lee S-H, Kim Y-S, Park OK, Hörtensteiner S, Paek N-C. Delayed degradation of chlorophylls and photosynthetic proteins in Arabidopsis autophagy mutants during stress-induced leaf yellowing. J Exp Bot. 2014:eru008 65(14):3915-25

61. Jahns P, Holzwarth AR. The role of the xanthophyll cycle and of lutein in photoprotection of photosystem II. Biochim Biophys Acta. 1817;2012:182-93.

62. Goss R, Lepetit B. Biodiversity of NPQ. J Plant Physiol. 2015;172:13-32.

63. Zubik M, Luchowski R, Grudzinski W, Gospodarek M, Gryczynski I, Gryczynski Z, et al. Light-induced isomerization of the LHCIl-bound xanthophyll neoxanthin: Possible implications for photoprotection in plants. Biochim Biophys Acta. 1807;2011:1237-43.

64. Aiamla-Or S, Shigyo M, Ito S-I, Yamauchi N. Involvement of chloroplast peroxidase on chlorophyll degradation in postharvest broccoli florets and its control by UV-B treatment. Food Chem. 2014;165:224-31.

65. Strid $\AA$, Chow WS, Anderson JM. UV-B damage and protection at the molecular level in plants. Photosynt Res. 1994;39:475-89.

66. Moon YR, Lee MH, Tovuu A, Lee C-H, Chung BY, Park Y-I, et al. Acute exposure to UV-B sensitizes cucumber, tomato, and Arabidopsis plants to photooxidative stress by inhibiting thermal energy dissipation and antioxidant defense. J Radiat Res. 2011;52:238-48.

67. Berkelaar EJ, Ormrod DP, Hale BA. The Influence of Photosynthetically Active Radiation on the Effects of Ultraviolet-B Radiation on Arabidopsis thaliana. Photochem Photobiol. 1996:64:110-6.

68. Götz M, Albert A, Stich S, Heller W, Scherb H, Krins A, et al. PAR modulation of the UV-dependent levels of flavonoid metabolites in Arabidopsis thaliana (L.) Heynh. leaf rosettes: Cumulative effects after a whole vegetative growth period. Protoplasma. 2010;243:95-103.

69. Cominelli E, Gusmaroli G, Allegra D, Galbiati M, Wade HK, Jenkins Gl, et al. Expression analysis of anthocyanin regulatory genes in response to different light qualities in Arabidopsis thaliana. J Plant Physiol. 2008;165:886-94.

70. Ryan KG, Swinny EE, Markham KR, Winefield C. Flavonoid gene expression and UV photoprotection in transgenic and mutant Petunia leaves. Phytochemistry. 2002;59:23-32.

71. Wingler A, Brownhill E, Pourtau N. Mechanisms of the light-dependent induction of cell death in tobacco plants with delayed senescence. J Exp Bot. 2005;56:2897-905.

72. Danon A, Rotari VI, Gordon A, Mailhac N, Gallois P. Ultraviolet-C overexposure induces programmed cell death in Arabidopsis, which is mediated by caspase-like activities and which can be suppressed by caspase inhibitors, p35 and Defender against Apoptotic Death. J Biol Chem. 2004;279:779-87.

73. Zapata J, Guera A, Esteban-Carrasco A, Martin M, Sabater B. Chloroplasts regulate leaf senescence: Delayed senescence in transgenic ndhF-defective tobacco. Cell Death Differ. 2005;12:1277-84.

74. Kim C, Meskauskiene R, Zhang S, Lee KP, Ashok ML, Blajecka K, et al. Chloroplasts of Arabidopsis are the source and a primary target of a plantspecific programmed cell death signaling pathway. Plant Cell. 2012;24:3026-39.

75. Gutiérrez J, González-Pérez S, García-García F, Daly CT, Lorenzo Ó, Revuelta $J$, et al. Programmed cell death activated by Rose Bengal in Arabidopsis thaliana cell suspension cultures requires functional chloroplasts. J Exp Bot 2014;65:3081-95. 